\title{
Especial
}

\section{Factores y riesgos laborales psicosociales: conceptualización, historia y cambios actuales}

\author{
Factors and occupational psychosocial risks: concept, history and current changes
}

\section{Bernardo Moreno Jiménez}

Departamento de Psicología Biológica y de la Salud. Facultad de Psicología. Universidad Autónoma de Madrid. España.

\author{
Correspondencia: \\ Bernardo Moreno Jiménez. \\ Facultad de Psicología \\ Universidad Autónoma de Madrid \\ C/ Ivan Pavlov, 6 \\ 28049 Madrid. España. \\ Tfno.: +34 914975185 \\ E-mail: bernardo.moreno@uam.es
}

Resumen

El trabajo ha sido históricamente un riesgo para la salud. Las condiciones laborales han supuesto habitualmente una amenaza a la salud que han ocasionado accidentes y enfermedades relacionadas con la salud de todo tipo. La imagen popular 1 asociada al trabajo ha sido claramente negativa. Los tiempos han cambiado de forma muy importante, pero las condiciones laborales siguen siendo preocupantes. La preocupación por los riesgos laborales se ha centrado históricamente en los riesgos físicos y ambientales, pero se ha producido una atención creciente en los riesgos psicosociales que exigen un mayor esfuerzo de definición en sus diferentes formas. En los tiempos actuales, debido a la expansión del mercado de servicios y a la globalización los riesgos psicosociales se han incrementado e intensificado. Los datos actuales muestran que sus efectos sobre la salud son amplios e importantes. Por ello, una atención integral a la salud laboral necesita cuidar de forma especial atención a los factores y riesgos psicosociales.

Palabras Clave: riesgos laborales, salud laboral, riesgos psicosociales, mercado de servicios, globalización.

\begin{abstract}
Work has been historically a health risk. Working conditions have usually been a threat to health causing accidents and many kind of health-related diseases. The popular image associated with work has clearly been negative. Times have changed very significantly, but working conditions remain a concern. Concern about occupational risks has historically focused on environmental and physical risks, but there has been increasing attention on psychosocial risks which require a greater effort to be defined. In modern times, due to services market expansion and globalization, psychosocial risks have increased and intensified. Current data show that psychosocial risks cause important health effects. Therefore, it is important make special attention to psychosocial factors and risks.
\end{abstract}

Key words: Occupational risks, Occupational Health, psychosocial risks, services market, globalization. 


\section{EL TRABAJO COMO RIESGO}

El Trabajo humano tiene elementos paradójicos: puede llevar a las personas a la excelencia o puede hacerles un daño inmenso a su salud, tanto física como psicológica y mentalmente; es algo que siempre se ha sabido. La historia está henchida de hechos y modelos que apoyan un aspecto y su opuesto ${ }^{1}$.

El trabajo físico, la mano de obra obligada, no ha sido bien vista a lo largo de la historia. Carente de cualquier protección, el trabajo era una forma de subsistencia que comprometía la salud de quienes no tenían más remedio que asumirlo. En el esclavismo, en el viejo sistema de producción oriental, el concepto de condiciones de trabajo y riesgo carecía de sentido. Carecía de valor el mismo trabajo físico que era considerado servil y sin valor $^{2}$, y la posible mala salud derivada era un problema individual de quienes trabajaban. El trabajador, quien tenía que vivir de su trabajo, era alguien desdeñable, su salud también ${ }^{3}$.

El panorama laboral ha cambiado drásticamente ${ }^{4}$. La aparición de los primeros estados, del sindicalismo, del reconocimiento de los derechos humanos y de las formas modernas de producción empresarial ha disminuido la dureza, frecuentemente inhumana, de las condiciones de trabajo. Las condiciones laborales han mejorado sustantivamente en los aspectos físicos del trabajo tales como horas de trabajo, sobreesfuerzos físicos, condiciones de temperatura, de ruido, ambientales y de higiene ${ }^{5}$. El efecto de tales modificaciones ha sido una disminución de la morbilidad y de la siniestralidad en el trabajo. El número de enfermedades laborales y de accidentes se ha controlado parcialmente y ha disminuido su incidencia. Pero probablemente, la mayor modificación se ha producido en el cambio del concepto de salud laboral que ha dejado de ser un problema individual para convertirse en un problema social y empresarial, y, principalmente, en un derecho del trabajador (Ley de Prevención de Riesgos Laborales 31/1995).

Históricamente, la atención sobre la amenaza a la salud proveniente del trabajo se ha centrado en los riesgos físicos, químicos y ambientales. Los daños directos más inmediatos a la salud suelen provenir de tales factores, causantes en su mayor parte de los accidentes y las enfermedades laborales o relacionadas con el trabajo. Es por ello que la legislación europea, la mundial en general, se ha ocupado principalmente de tales factores mediante la legislación adecuada que trata de prevenir y controlar tales riesgos. No en vano la primera legislación estuvo centrada en los accidentes ${ }^{6}$.

Los logros son parciales. En primer lugar, se han producido principalmente en el occidente laboral, en los países desarrollados como lo son los países integrantes de la OCDE que aúna a los países más ricos y prósperos de la Tierra. En muchos otros países, las condiciones laborales son ampliamente deficitarias y reproducen formas similares a las condiciones laborales insalubres superadas en el Occidente laboral. En segundo lugar, en los mismos países de la OCDE, siguen perviviendo condiciones laborales inadecuadas. La Organización Internacional del Trabajo (OIT) ha desarrollado el concepto de "decent work" (trabajo decente) para referirse al contexto laboral básico del que debe partirse en cualquier parte del mundo. (http://www.wageindicator.org/main/decent-work-check).

El concepto de "Trabajo decente" incluye aquellos aspectos laborales que hacen referencia tanto al derecho a la salud en el trabajo como un derecho básico como al derecho social al trabajo en sí mismo, dos aspectos vinculados mutuamente y que no pueden desarrollarse el uno al margen del otro (http://www.ilo.org/global/about-the-ilo/ decent-work-agenda/lang--en/index.htm). A lo largo de la historia, e incluso en la actualidad, no ha sido así. En los tiempos de la primera industrialización, se encontraba trabajo con facilidad si se aceptaba cualquier condición de trabajo, salarial y ambiental ${ }^{7,8}$.Algo semejante ocurre en los tiempos de crisis económicas en los que la accesibilidad al trabajo disminuye y las condiciones del mismo empeoran ${ }^{9}$. Estrictamente, es una consecuencia de la aplicación de las leyes del mercado sobre la oferta de trabajo. 


\section{LOS RIESGOS LABORALES}

Los riesgos laborales han ido cambiando a lo largo de toda la historia. El mismo concepto de riesgo laboral supone un logro porque durante mucho tiempo ha carecido de sentido. El trabajador carecía de cualquier derecho; su trabajo era su vida y su obligación, se le mantenía para que trabajara, y si sufría cualquier daño, parcial o letal, era su suerte. Objetivo del trabajo era obtener unos resultados en el menor tiempo, al margen de los costes, especialmente de los humanos.

El concepto de riesgo laboral para la salud aparece con el reconocimiento, implícito o explicito, del derecho a la integridad física y a la salud, algo que aparece en la Alta Edad Media y en el Renacimiento con la aparición y desarrollo de los gremios y la preocupación por el buen hacer y la experiencia adquirida por lo trabajadores artesanos. La experiencia y la valían adquieren un valor al mismo tiempo que en las ciudades disminuye la subordinación a los señores. Los gremios, primera aproximación a los sindicatos, suponen la primera defensa formal contra determinadas condiciones laborales y trato a los trabajadores. El riesgo laboral contra la salud aparece definido como las situaciones y conductas que no pueden ser aceptadas por sus nocivas consecuencias para los trabajadores.

La Revolución francesa (1789), la aparición de los sindicatos (S. XIX), el reconocimiento formal de los Derechos Humanos por las Naciones Unidas (1948), la constitución de los estados democráticos posteriores a la Segunda Guerra mundial, suponen indirectamente hitos en la conceptualización de la salud laboral que se origina principalmente en el reconocimiento del derecho del trabajador a su integridad física, mental y social. La salud laboral como derecho no es el resultado ni de la Medicina del Trabajo ni de la Psicología del Trabajo, sino del reconocimiento de que los trabajadores no pueden ser expuestos a situaciones que menoscaben su salud. Hasta cierto punto, la función de ambas ha sido más bien negativa pues, de hecho, uno de sus objetivos más explícitos consistía en delimitar hasta que límites se podía llegar en las condiciones de trabajo sin que afectara claramente a la salud y al rendimiento de los trabajadores.

En Europa, la preocupación por la prevención de riesgos laborales se activa después de la Segunda Guerra Mundial y va de la mano de la elaboración de los diferentes tratados de la constitución de Europa. En 1989 aparece la Directiva Marco Europea de prevención de riesgos laborales centrada en el concepto de condiciones laborales, su evaluación, su control y su prevención (89/391/CEE), pero que amplía el concepto de condiciones laborales, incluyendo la salud como objeto final de la Directiva. Sin embargo, sigue centrada en los riesgos físicos, químicos, ambientales, dado que los daños directos más inmediatos a la salud suelen provenir de tales factores, causantes en su mayor parte de los accidentes y las enfermedades laborales. Es un dato que la legislación europea, la mundial en general, se ha ocupado principalmente de tales factores mediante una legislación que trata de prevenir y controlar tales riesgos.

En España, el planteamiento actual sobre los riesgos laborales se origina en la Ley de Prevención de Riesgos laborales (31/1995), resultante de la transposición de la Directiva Marco Europea. La Ley de Prevención de Riesgos Laborales no aborda en ningún momento los riesgos psicosociales, en realidad, no aparece en ella ninguna referencia a riesgos específicos, pero sí establece de forma taxativa en su artículo 12 parágrafo 2 la obligación del empresario de "garantizar la seguridad y la salud de los trabajadores a su servicio en todos los aspectos relacionados con el trabajo", lo que incluye a los factores relacionados con la organización del trabajo.

Es en el Real Decreto 39/1997 de los Servicios de Prevención, en el anexo VI, en el que describiendo la formación necesaria para el Técnico Superior En Prevención de Riesgos Psicosociales se explicita como una de las especialidades la de Ergonomía y Factores Psicosociales. Como tal, es probablemente la primera referencia legal a los factores psicosociales. Cuando posteriormente los escasos manuales de Ergonomía y Psicosociología aplicada exponen el tema ${ }^{10,11}$ se centran en temas tales como el contenido del trabajo, la carga laboral, el control de la tarea, la supervisión y las relaciones laborales. 


\section{FACTORES PSICOSOCIALES, FACTORES PSICOSOCIALES DE RIESGO Y RIESGOS PSICOSOCIALES}

En la actualidad, en la literatura sobre el tema existen tres formas de referirse a los aspectos psicosociales: como factores psicosociales, como factores psicosociales de riesgo y como riesgos psicosociales. Frecuentemente se usan como intercambiables y no existe una diferenciación usual y académica neta entre ellos, sin embargo se pueden advertir aspectos diferenciales en su uso y una cierta gradación en las connotaciones implicadas. Quizás sea oportuno tratar de matizarlas.

El concepto de factores psicosociales en el trabajo es relativamente reciente y probablemente se origina en el último cuarto de siglo pasado. Es a partir de entonces cuando, de forma muy escasa, se comienza a hablar de ellos. Una de las primeras referencias oficiales al tema aparece en 1984 en el trabajo "Los factores psicosociales en el trabajo: reconocimiento y control" en un documento publicado por la Organización Internacional del Trabajo. Desde el primer momento de su formulación se insiste en la importancia de sus efectos reales, en la dificultad de su formulación objetiva y en su complejidad. "Los factores psicosociales en el trabajo son complejos y difíciles de entender, dado que representan el conjunto de las percepciones y experiencias del trabajador y abarcan muchos aspectos" ${ }^{12}$. Las primeras listas de riesgos psicosociales son amplias y abarcan gran cantidad de aspectos: la sobrecarga en el trabajo, la falta de control, el conflicto de autoridad, la desigualdad en el salario, la falta de seguridad en el trabajo, los problemas de las relaciones laborales y el trabajo por turnos ${ }^{12}$. Llama la atención que la práctica totalidad del listado siga siendo actual.

A partir de entonces, el concepto de factores psicosociales laborales y de factores organizacionales laborales se asocian y tienden a intercambiarse tal como expone la tercera edición de la Enciclopedia de la Seguridad y La Salud en el Trabajo ${ }^{13}$. Ambos expresan la importancia que tienen los factores sociales en la conducta y en la salud de los trabajadores, positiva y negativamente.

Lamentablemente, se ha insistido, de hecho, mucho más en los factores psicosociales negativos que en los positivos, en los riesgos que en el desarrollo, cuando los factores psicosociales $\mathrm{u}$ organizacionales pueden tener tanto repercusiones negativas como positivas. La perspectiva legal, que puede ser obvia, parece que debe centrarse en la evitación del daño, en la obligación de no causar lesiones a la salud del trabajador, pero la perspectiva conceptual y organizacional debería haber atendido tanto a las repercusiones negativas como a las positivas. Es probable que el desarrollo de una perspectiva positiva de los factores psicosociales laborales hubiera facilitado pensar el gasto como inversión y desarrollo de la seguridad y salud laboral, favoreciendo el desarrollo de organizaciones saludables $^{14}$.

En este sentido, parece que debería diferenciarse entre los factores psicosociales y los factores psicosociales de riesgo. Los primeros son descriptivos, aluden a la estructura organizacional $^{15}$, a las condiciones psicosociales del trabajo como la cultura corporativa, el clima laboral, el estilo de liderazgo o el diseño del puesto de trabajo, factores que como tales pueden ser positivos o negativos ${ }^{16}$, los segundos son predictivos, se refieren a las condiciones organizacionales cuando tienen una probabilidad de tener efectos lesivos sobre la salud de los trabajadores, cuando son elementos con probabilidad de afectar negativamente la salud y el bienestar del trabajador ${ }^{17}$, cuando actúan como factores desencadenantes de la tensión y el estrés laboral.

Desde esta perspectiva, los factores psicosociales de riesgo son factores probables de daño a la salud, son negativos y pueden afectar tanto a la salud física como a la psicológica $^{18}$. Son factores de estrés que pueden alterar y desequilibrar los recursos y las capacidades de la persona para manejar y responder al flujo de la actividad derivada del trabajo $^{19}$. Son innumerables y pueden provenir de los múltiples componentes del trabajo: falta de control en el trabajo, muchas horas de trabajo, intensidad del ritmo de trabajo, horarios cambiantes e imprevisibles, mala comunicación organizacional ascendente, 
horizontal o descendente, ambigüedad o sobrecarga de rol y otros muchos. Dado que pueden originarse en cualquiera de los múltiples desequilibrios entre demandas y recursos, la lista es abierta y prácticamente interminable. Los principales modelos de estrés laboral ${ }^{20,21}$ han expuesto los mecanismos principales que dan lugar a los factores de estrés laboral y la investigación psicofisiológica, médica y psicológica ha propuesto diferentes tipos de procesos que relacionan los factores de estrés laboral y el daño a la salud $^{22,23,24,25,}$ tanto física como psicológica.

Los factores psicosociales de riesgo son factores reales de riesgo que amenazan la salud de los trabajadores, pero tienen características propias que hacen más difícil su manejo, evaluación y control. Entre las más importantes de ellas podrían citarse las siguientes: 1) Se extienden en el espacio y el tiempo, 2) Son difíciles de objetivar, 3) Afectan a los otros riesgos, 4) Tienen escasa cobertura legal, 5) Están moderados por otros factores, 6) Son difíciles de modificar. Todas estas características dificultan su prevención, evaluación y control.

En los últimos años, probablemente a partir de la primera década de este siglo xxI, se ha comenzado, de hecho, a utilizar otra terminología y otra conceptualización ligeramente diferente pero de una gran importancia conceptual: los riesgos psicosociales. Cada vez con mayor frecuencia se habla directamente de los riesgos psicosociales para referirse a una serie de situaciones de gran peso en la vida laboral que pueden afectar gravemente la salud de los trabajadores. Aunque la delimitación entre los factores psicosociales de riesgo y los riesgos psicosociales no siempre resulta clara, existe una tendencia a reconocer como riesgos psicosociales a situaciones como la violencia y agresión en el trabajo, el acoso laboral y sexual, el estrés laboral crónico, el burnout o desgaste profesional, y la inseguridad contractual. A ellos pueden añadirse otros. www. insht.es/InshtWeb/.../factores\%20riesgos\%20psico.pdf.

Los riesgos psicosociales laborales son situaciones laborales que tienen una alta probabilidad de dañar gravemente la salud de los trabajadores, física, social o mentalmente. Los riesgos psicosociales laborales son situaciones que afectan habitualmente de forma importante y grave la salud. Mientras que los factores de riesgo psicosocial son habitualmente factores con diferentes niveles de probabilidad de ocasionar daños de todo tipo, los riesgos psicosociales tienen una alta probabilidad de generar consecuencias principalmente graves.

Entre los factores psicosociales y los riesgos psicosociales hay otras diferencias importantes, como que los riesgos psicosociales afectan a los derechos fundamentales del trabajador tales como han sido recogidos en la mayoría de constituciones nacionales actuales, y que por lo mismo tienen formas de protección legal, tanto a nivel nacional como internacional. Ejemplos de ello son las directivas a nivel europeo "Framework Agreement on Work Related Stress" ${ }^{26}$ y la "Framework Agreement on Harassment and Violence at Work" ${ }^{27}$ que sugieren posibles bases legales europeas. Como respuesta a tales iniciativas, diferentes países europeos han procedido a elaborar legislaciones nacionales específicas referidas estrictamente a los riesgos psicosociales ${ }^{28}$.

Otras diferencias entre ambos consisten en la gravedad y globalidad de las consecuencias de los riesgos psicosociales, lo que entraña repercusiones que van desde los aspectos físicos y psicológicos hasta los mentales. No es extraño que los riesgos psicosociales tengan consecuencias mentales graves, que alteren el equilibrio mental de la persona con afecciones claramente psicopatológicas o psiquiátricas. Un ejemplo claro es el estrés postraumático, prácticamente inexistente en los factores psicosociales de riesgo, pero nada extraño en los riesgos psicosociales como las situaciones de violencia y de acoso laboral o sexual. Debido a la importancia de los resultados, las variables mediadoras, aunque siempre presentes, tienden a tener menos importancia. Es decir, en las situaciones graves, los efectos principales son dominantes, mientras que en las situaciones leves o menores, la influencia de los efectos mediadores pueden ser decisiva en el tamaño del efecto final, en las consecuencias. 
Estas diferencias son la probable razón de que se haya producido recientemente una acentuación de los riesgos psicosociales. Llaman más atención, especialmente la mediática. No pocos de los riesgos psicosociales han sensibilizado a la población por sus efectos considerables, conducentes en algunos al suicidio por ejemplo o a traumas importantes. El acoso y la violencia laboral han sido algunos de los más mencionados y referidos. Sin embargo, aún reconociendo que su tasa ha podido aumentar y que las consecuencias que suelen derivarse de ellos son habitualmente importantes, no deberían desplazar la atención y la preocupación por los factores psicosociales de riesgo. Entre ellos y los riesgos psicosociales hay incluso una relación de facilitación. Un clima laboral autoritativo y desconsiderado es un factor psicosocial de riesgo ${ }^{29}$, pero es también el marco propicio para que aparezcan riesgos psicosociales como el abuso, la violencia y al acoso. En este sentido, la prevención comienza al nivel de los factores psicosociales de riesgo.

Incluso la atención exclusiva a los riesgos psicosociales puede ser un indicador de la pérdida de capacidad de análisis de la salud laboral. De la misma forma que no se puede atender sólo a los accidentes mortales, sino que la verdadera prevención comienza en la atención a los incidentes blancos laborales que no producen lesiones, la prevención psicosocial comienza en el análisis de los factores de riesgo psicosocial. Atender sólo a los riesgos psicosociales supone dirigir la atención sólo hacia las manifestaciones más graves, descuidando su génesis. Los factores psicosociales de riesgo son factores con consecuencias nocivas para la salud laboral ${ }^{30}$ que no pueden ser desatendidos.

\section{ATENCIÓN Y SEGUIMIENTO DE LOS FACTORES Y RIESGOS PSICOSOCIALES}

A partir de 1987 el Instituto Nacional de Seguridad e Higiene en el Trabajo (INSHT) comenzó a aplicar "Encuestas nacionales de condiciones de trabajo" y a partir de la tercera encuesta ${ }^{31}$ incluye elementos de análisis psicosocial referidos a los contenidos del estatus del puesto, del horario de trabajo y la participación laboral, categorías de análisis de análisis que se han mantenido con variaciones en las sucesivas ediciones. La serie de Notas Técnicas de Prevención (NTP) comienza a partir de 1988 a incluir contenidos psicosociales.

En la Unión Europea, la Fundación para la Mejora de las Condiciones de Vida realiza cada cinco años una encuesta sobre las Condiciones de trabajo en Europa. En la primera encuesta de 1991 incluía como aspectos psicosociales la información, el entrenamiento y el apoyo social. A partir de la segunda encuesta (1996), el tema se amplía organizándose en dos grandes apartados, uno organizacional que incluye tiempo de trabajo, ritmo, control, autonomía, contenido del trabajo y salario y otro apartado de tipo social que contiene aspectos como participación, igualdad de oportunidades y violencia. Es a partir de la tercera edición del 2000 cuando se incluye de forma específica los contenidos referidos a la violencia, al acoso laboral y al acoso sexual, sección que se mantiene con ligeras modificaciones en la edición de 2005. En la última encuesta, efectuada en 2010, se puede observar un marcado desplazamiento de la encuesta en su totalidad hacia un enfoque organizacional y psicosocial. La tabla 1 expone las categorías consideradas y el número de cuestiones por cada una de ellas.

Tabla 1. Encuesta EWCS 2010. Categorías de análisis y $\mathbf{n} .^{\circ}$ de cuestiones

\begin{tabular}{lrlr}
\hline Contexto de trabajo & 11 & Tiempo de trabajo & 12 \\
Intensidad del trabajo & 9 & Factores Físicos & 12 \\
Factores cognitivos & 9 & Factores psicosociales & 7 \\
Salud y bienestar laboral & 6 & Formación, Desarrollo carreras & 8 \\
Organización del trabajo & 9 & Relaciones sociales & 5 \\
Satisfacción laboral & 7 & Familia-trabajo, seguridad economía & 7 \\
Violencia, acoso, discriminación & 5 & & \\
\hline
\end{tabular}


El análisis de la Encuesta Europea 2010 parece indicar que se ha producido un desplazamiento de los factores físicos y ambientales hacia aspectos organizacionales, sociales, psicológicos y personales. Como factores psicosociales incluye específicamente los aspectos emocionales asociados al trabajo, como el trabajo con atención al cliente. La sección dedicada a la violencia ha omitido los aspectos referidos al acoso sexual y a la atención sexual no deseada. Con todo, la categorización de las secciones incluye en algunos casos contenidos claramente diferentes.

Algo semejante ocurre con los datos españoles aportados principalmente por el INSHT y las encuestas que ha venido realizando periódicamente. Es en la tercera encuesta nacional (1999) cuando se incorpora por primera vez un apartado dedicado a los factores psicosociales, pero ha sido en la VI Encuesta Nacional $^{32}$ en la que se ha atendido de forma más completa a los factores y riesgos psicosociales, incluyendo en el apartado 10 aspectos tales como inseguridad laboral, conflicto trabajo-familia, violencia física, amenazas de violencia y conductas de acoso.

Los datos expuestos indican probablemente que, al menos al nivel europeo, se ha producido un desplazamiento en la atención de los riesgos laborales hacia aquellos de tipo organizacional, social, psicológico, familiar y personal, probablemente como resultado de una triple tendencia: la mejora de las condiciones físicas, ambientales y ergonómicas del trabajo, los cambios organizacionales del trabajo y el desarrollo del trabajo terciario, dedicado a los servicios y la atención a las personas.

\section{CAMBIOS DEL MUNDO DEL TRABAJO}

Los cambios vividos en nuestra sociedad moderna provienen en gran medida de dos fuentes principales: los estados democráticos y las empresas actuales. Sin estados democráticos atentos a los derechos y exigencias de la sociedad, equilibradores de las diferencias de poder, del oligopolio y la plutocracia, es difícil pensar el desarrollo económico y social en general $^{33,34}$.

Los estados democráticos han dado lugar a un estado de bienestar y desarrollo social sin parangón en la historia ${ }^{35,4}$. El desarrollo del estado del bienestar ha supuesto la aparición de un espacio social y público de posibilidades y de libertades que ha incluido al mundo del trabajo, y las condiciones de trabajo reconocidas exigibles.

Los nuevos estados democráticos se caracterizaron en sus comienzos por su oposición a los regímenes despóticos y autoritarios del fascismo, nazismo, comunismo y el régimen de vida que imperaba en ellos dominado por la inseguridad y el miedo, cuando no el terror. La aparición y desarrollo en los nuevos estados de la sociodemocracia en la década de $1960^{4}$, especialmente en los estados escandinavos, instauró la consolidación del "hombre público" ${ }^{36}$, sujeto y protagonista de la acción pública, capaz de transformar los derechos formales legales en derechos reales materiales en su propio estado-nación y ciudad. Es el resultado de la acción pública y política.

Por la otra parte, la empresa, la libre iniciativa y su capacidad de innovación y desarrollo, ha dado lugar a un auge económico, comercial y tecnológico sin precedentes. Su inserción en el tejido social ha cambiado el panorama de la vida moderna y las formas de vida, dándoles una capacidad de variación, de posibilidades y facilidades nunca alcanzado hasta el momento. Sin la empresa es imposible comprender y explicar el desarrollo logrado, la vida real contemporánea en sus aspectos comerciales, sociales, económicos, tecnológicos e incluso la identidad social postmoderna ${ }^{37,38}$. La postmodernidad no es sólo el resultado de las nuevas ideologías sino también de la nueva configuración postindustrial ${ }^{39,40}$ en nuestras sociedades.

Probablemente la primera característica relevante de la empresa moderna es su dinamismo, variabilidad y capacidad de cambio. Como dato y como modelo, la empresa flexible se ha convertido en aspiración e ideal ${ }^{41}$. La flexibilidad capacita a la empresa para 
responder a las variaciones continuas del mercado en las fluctuaciones de la demanda, en la aparición de nuevos productos, en la respuesta a nuevas tecnologías y competencias. Pero esa misma flexibilidad de la empresa como organización ha conducido inevitablemente al "trabajo flexible" ${ }^{42}$, que conlleva inevitablemente la inseguridad y la incertidumbre del mismo mercado, del trabajo y del trabajador ${ }^{43}$. La misma empresa flexible acaba produciendo ella misma las turbulencias a las que debería responder. La empresa como tal se convierte en factor de riesgo para el mercado, para la economía, y lo que es más importante, para el mismo trabajador.

La empresa moderna ha recorrido un largo camino desde la revolución industrial hasta el momento actual ${ }^{44,45}$. El imaginario popular que asocia el trabajo a las condiciones más abusivas y denigrantes de las primeras explotaciones industriales en el siglo xviII, ha vinculado con frecuencia el trabajo con la barbarie, la mano de obra barata, las largas horas de trabajo y el esfuerzo manual sin fin. Sin embargo, el mismo éxito y desarrollo de la empresa, sus logros productivos y comerciales la han obligado a transformarse internamente y cambiar desde dentro su propia organización. A lo largo de ese recorrido se encuentra lo mejor y lo peor: la excelencia y el descaro oportunista cuando no mezquino. Ese recorrido de luces y sombras es el mismo que presenta el panorama de la situación actual de las empresas: contradictorio y con alternativas diversas y opuestas.

En el lado organizacional más positivo, las organizaciones no sólo han descubierto el valor de los trabajadores como recursos humanos ${ }^{46}$ y lo que significa la experiencia adquirida en el trabajo y en la empresa, sino que ha valorizado su lado más humano como persona. El descubrimiento organizacional más hondo ha consistido en la consideración de que la gestión de los recursos humanos más eficiente es su consideración como personas ${ }^{47}$. Lo que facilita la gestión estratégica del capital humano es el supuesto básico de que los trabajadores son personas activas, agentes de su propio comportamiento ${ }^{48}$. Como ha recordado Sennet, ${ }^{49}$ uno de los sociólogos del trabajo actuales más relevantes, ello conlleva la recuperación del respeto como categoría básica de las relaciones laborales.

Ha sido el descubrimiento de esta perspectiva, o la revalorización de lo que siempre estuvo ahí, aunque olvidado, lo que ha dado lugar a formulaciones empresariales que plantean la organización como un sistema de producción e intercambio de experiencia y conocimientos $^{50}$, e incluso que revalorizan el complejo mundo emocional como una frontera más del funcionamiento empresarial, con todas sus luces y sombras ${ }^{51,52}$. Desde esta perspectiva, el primer planteamiento del recurso humano como prolongación instrumental, se convierte realmente en capital intelectual y finalmente en capital psicológico $^{53}$. Es este desarrollo el que ha posibilitado toda una andadura positiva, conceptual y empírica, en las relaciones entre la empresa y las nuevas condiciones organizacionales ${ }^{54}$. Sin este planteamiento no es posible entender el "comportamiento organizacional" actual en su complejidad y realidad ${ }^{55,56}$.

Incluso desde el punto de vista más funcional, es decir desde el punto de vista de la productividad, del logro de los objetivos empresariales e incluso de la obtención de los beneficios económicos buscados, las organizaciones laborales han hecho un largo recorrido desde el primer industrialismo y las formulaciones tayloristas y fordistas ${ }^{57}$ hasta los planteamientos actuales. Algunos de los desarrollos más importantes en este contexto han sido los de calidad de vida, responsabilidad social corporativa y la acción social de las empresas.

Los modelos de calidad de vida laboral han mostrado que la calidad del producto está inevitablemente asociada a la calidad de vida laboral, es decir que la calidad de los resultados y del sistema de producción está vinculada con la calidad del sistema de vida laboral ${ }^{58}$. Otro logro del modelo ha consistido en el reconocimiento de que la calidad de vida laboral no puede ser evaluada exclusivamente por elementos subjetivos y emocionales, como la satisfacción laboral, sino que es necesaria la búsqueda de marcadores objetivos ${ }^{59}$.

El planteamiento de la Responsabilidad Social Corporativa ha ido más allá de la simple mejora de los sistemas de producción internos de la empresa y se ha planteado una relación global con el marco ambiental de su enclave geográfico, con el contexto 
social y político de su ubicación sociodemográfica ${ }^{60-62}$. Finalmente, el modelo de empresas de acción social se propone objetivos que no son estrictamente lucrativos o financieros sino de mejora de las condiciones reales de una sociedad y una población con limitaciones y necesidades específicas ${ }^{63,64}$.

En su globalidad, este tipo de planteamientos es lo que ha permitido hablar de organizaciones saludables ${ }^{65}, 14$, organizaciones que cumplen sus objetivos comerciales y económicos y facilitan el desarrollo profesional y personal de sus trabajadores. Actualmente, los listados de empresas como "Great places to work" y "Covalence EthicalQuote Ranking” introducen parámetros comparativos del buen funcionamiento de las empresas que incluyen no sólo ventas y beneficios, sino otros criterios laborales más amplios como el bienestar de los trabajadores y valores éticos, lo que obliga a las empresas a un esfuerzo por la excelencia real a fin de mantener una imagen corporativa presentable.

Junto a estas luces, las sombras de las empresas no son pocas y menos en los últimos tiempos. La evolución y cambio de las empresas se está produciendo en todos los campos: organizacionales, tecnológicos, comerciales y económicos entre los principales. En todos ellos, el cambio supone un esfuerzo de adaptación y una respuesta de estrés. La aceleración de la historia causa desequilibrios y produce nuevos riesgos, precisamente por ello nuestra sociedad actual ha sido conceptualizada como "La sociedad del riesgo" "66, 67, una sociedad dominada por relaciones y situaciones sobre las que se tiene escaso control, lo que lleva a una generalización de la inseguridad, del miedo y del estrés.

\section{LOS NUEVOS RIESGOS PSICOSOCIALES EMERGENTES}

Resultado de todo ello es una preocupación global por la aparición de nuevos riesgos de toda índole. En el marco laboral preocupan especialmente los llamados riesgos emergentes ${ }^{68}$. Este tipo de riesgos provienen de múltiples campos, como las nuevas tecnologías, los nuevos sistemas de producción, las nuevas materias primas y los nuevos compuestos químicos y biológicos. En este apartado una preocupación especial la suscita el mundo cambiante de las organizaciones, las fluctuaciones económicas rápidas, los nuevos sistemas de trabajo, la nueva sociodemografia del mercado laboral, las nuevas formas de contratación, y en general, las nuevas formulaciones de las relaciones laborales, es decir lo que se ha denominado "riesgos psicosociales emergentes" 69,70

El Estudio de la Agencia Europea para la Seguridad y la Salud en el Trabajo sobre los riesgos psicosociales emergentes ${ }^{69}$, estableció una lista de 42 riesgos psicosociales. Gran parte de estos nuevos riesgos psicosociales están causados por un doble fenómeno: la expansión del sector terciario de la producción, el sector de servicios, y el proceso de la globalización actual en sus múltiples facetas, una de las cuales es la del mercado de trabajo. Ambos procesos están estrechamente asociados.

El sector servicios del mercado laboral ha aumentado vertiginosamente desde la mitad del siglo xx a la actualidad. Tal como ha descrito una obra de referencia básica en Ciencias Sociales "El advenimiento de la sociedad Postindustrial" ${ }^{11}$, la economía actual se ha desplazado "de los bienes a los servicios" creando un nuevo y expansivo mercado de trabajo que no tiene las mismas condiciones de trabajo que tenía y tiene el sector extractivo o el industrial o manufacturero. El empleo industrial alcanza su apogeo hacia 1970 y a partir de ese momento la mayor parte de los estados europeos, de forma diferente y con diferentes tipos de servicios, entra en un ciclo dominado por la expansión del mercado de servicios, lo que supone un cambio importante en el marco social y material del empleo.

En España, ${ }^{72}$ el cambio se produce en la mitad de la década de los ochenta, en particular de los servicios públicos como consecuencia de la reforma y descentralización de la Administración Pública y como consecuencia de la expansión del estado de bienestar, centrado en la administración y en la sanidad. En el año 2006, dos terceras partes del mercado de trabajo en España, el 65,7\%, estaban en el mercado laboral de servicios ${ }^{73}$. 
Los riesgos laborales del mercado de servicios son principalmente psicosociales. Son riesgos que provienen de las nuevas formas de trabajo, del dinamismo de las nuevas organizaciones y sus formas cambiantes. Son principalmente riesgos que provienen de las múltiples formas de atender a una clientela en interacción dinámica con el trabajador. Aunque la interacción social siempre ha sido una característica del trabajo, la novedad consiste en que se ha convertido en su característica central.

Entre los riesgos de tipo interactivos la violencia, el acoso y el acoso sexual tienen tasas particularmente altas en el sector servicios, incluso algunos de ellos se dan forma particular en este sector. Ocurre por ejemplo en la violencia de Tipo II o violencia del cliente. Ocurre igualmente de forma particular en el riesgo del acoso sexual debido al establecimiento usual de formas de relación más próximas y en ambientes más restringidos. Aunque tanto la violencia como el acoso sexual pueden ocurrir en contextos laborales generales, sus connotaciones son diferentes.

Algunos de los riesgos psicosociales propios del sector servicios provienen del cambio de rol del trabajador en la misma transacción laboral y comercial. Consiste en que algunas de las transacciones demandadas pueden afectar a aéreas de la propia identidad del trabajador. Lo que se puede solicitar en algunas transacciones laborales no es que el trabajador medie en la obtención de un bien u objetivo, como un café, sino que el objeto del servicio son características personales del trabajador, como su competencia, su dedicación y atención; es lo que ocurre en el área de la salud y la educación y en otras áreas.

El trabajo emocional ilustra este nuevo tipo de riesgos psicosociales. Un buen número de trabajos asistenciales y de servicios exigen hoy día la autorregulación de las emociones, es decir, la inhibición de las emociones negativas y la expresión forzada de emociones negativas. En un caso y otro, los efectos son de desgaste emocional, de disonancia emocional: el trabajador tiene que mostrar emocionalmente lo que no siente. La exigencia del trabajo emocional es tanto más importante mientras mayor es la necesidad de asegurarse la satisfacción del cliente. Tal como ha expuesto Hochschild ${ }^{74}$, la primera autora en hablar del tema explícitamente, el problema radica en la habitual conexión entre las emociones y las expresiones corporales y gestuales. El autocontrol emocional, positivo y negativo, supone un ejercicio continuo de autocontención corporal, de representar su rol, de actuar emocionalmente. No sólo se le pide un resultado y un servicio, sino que la ejecución del mismo sea placentera y agradable, que muestre emociones positivas por lo que está haciendo. Las normas organizacionales pueden en este sentido ser taxativas debido a que la misma imagen corporativa depende de la imagen emocional de sus trabajadores ${ }^{75}$. No se pide sólo un servicio, sino además un servicio emocionalmente reforzante para el cliente. El agotamiento resultante de esta constante actitud interna y externa puede resultar extenuante.

El trabajo emocional es representativo de algunos de los cambios que se están produciendo en el mercado laboral, y específicamente en el ámbito del mercado asistencial y de servicios consistente en la demanda por parte de la empresa de un esfuerzo íntegro de la persona que incluye los sentimientos que puede permitirse y debe mantener. El trabajo de servicios suele conllevar una demanda no sólo de tiempo, de dedicación y de esfuerzo sino de actitud ${ }^{76}$. Las consecuencias de ello es que los resultados de deterioro de la persona pueden ser fácilmente mayores, físicos ${ }^{77}$, y mentales $^{78}$.

\section{LA GLOBALIZACIÓN Y SUS EFECTOS}

El recurso a la globalización para explicar la realidad actual es cada vez más frecuente, pero no es banal. Los cambios que se están produciendo no suelen ser cambios aislados, puntuales, sino cambios que se producen en una totalidad, en un sistema. Si esto es válido en general, lo es todavía más estrictamente para el campo económico con el que comenzó todo el proceso. La atenuación de fronteras para los capitales, para el comercio, 
para el tránsito de materias primas y de personas, es el origen de todo lo que ha venido después, incluido el mundo del trabajo ${ }^{79}$.

En el ámbito de la Salud Laboral, los efectos de la globalización han sido muy heterogéneos. Mientras en los países en vías de desarrollo y en los países emergentes probablemente ha supuesto una mejora, parcial, de las condiciones materiales de trabajo, en los países occidentales, en general en los países de la OCDE, los efectos han sido perniciosos tanto por lo que respecta a las condiciones de trabajo como, especialmente en lo que concierne a la seguridad del empleo. Una conclusión clara que puede hacerse de forma global es que la globalización ha aumentado especialmente los riesgos psicosociales.

Los efectos de la globalización sobre la economía y el trabajo han sido múltiples y sumamente complejos ${ }^{80,81}$. Aunque hasta ahora, la globalización tenía sus defensores y detractores, la crisis económica actual que se viene arrastrando desde 2007 ha aumentado sus críticos y enfatizado sus aspectos más nocivos. Si antes afectaba a amplios grupos y comunidades, ahora son países enteros y el mismo sistema económico mundial el que parece haber entrado en crisis y en un ciclo de recesión. En los primeros momentos de la globalización la mirada se ponía en el nuevo tipo de empresas que estaban apareciendo, en su enorme magnitud y la dificultad de ponerle trabas a su acción mundial ${ }^{82,83}$. Las empresas multi y trans-nacionales imponían sus prácticas sobre la misma voluntad de algunos estados ${ }^{85}$ y obligaban a aceptar, especialmente en las zonas francas de producción, condiciones materiales inaceptables para un trabajo decente ${ }^{84}$.

Las estrategias organizacionales utilizadas para mantener su competitividad, cuando no su hegemonía, han ido desde la deslocalización de las empresas hacia mercados legalmente más desregulados, menos exigentes y más acomodaticios, o la utilización de estrategias de subcontratación o de outsourcing que facilitaba una disminución del coste total $^{85}$. Probablemente la operación estratégica más habitual ha consistido en las adquisiciones y fusiones de las empresas a fin de obtener el dominio del mercado, la supresión de la competencia, el control de los sistemas de producción y la capacidad de imponer el precio final de los productos ${ }^{86}$. Otro sistema habitual ha consistido en la disminución de plantillas (downsizing) para aumentar beneficios y reducir costes ${ }^{87}$. Las consecuencias de todas estas estrategias ha sido la aparición de nuevos riesgos emergentes organizacionales, la acumulación del riesgo global y el aumento de la siniestralidad ${ }^{88}$.

En los últimos cuatro años el panorama no ha perdido la gravedad descrita y ha acentuado un problema que ya estaba presente: la inseguridad contractual. Durante no pocos años la hegemonía en el mercado comercial y laboral estaba determinada por las grandes empresas que dictaban las políticas mundiales, sin embargo en los últimos años la hegemonía ha pasado a mano de los mercados económicos, especialmente financieros. Se ha producido un desplazamiento de los bancos comerciales tradicionales hacia los bancos de inversión que han centrado sus operaciones en las operaciones financieras a corto plazo $^{89,81}$. El resultado ha sido una pérdida de la fuerza de la economía real y del protagonismo de las empresas. La consecuencia más inmediata y global ha sido un hundimiento del mercado del trabajo y el aumento de la inseguridad laboral en todas sus formas, principalmente de la contractual ${ }^{90}$.

La inseguridad contractual es probablemente el primero y el más importante riesgo psicosocial actual en todo el mundo. Las tasas de paro en USA han alcanzado las cotas más altas conocidas, y lo mismo ha ocurrido en no pocos países de la OCDE. La consecuencia general ha consistido en un aumento del desempleo y un consecutivo empobrecimiento general. En España, país con un problema crónico de desempleo ${ }^{72}$, se ha convertido en el principal problema social, económico y político actual.

El trabajo de la OSHA ${ }^{69}$ sobre los nuevos riesgos psicosociales emergentes engloba cuatro de los diez riesgos más importantes en la categoría de inseguridad contractual, lo que indica su importancia básica. El problema afecta a tres grandes colectivos de trabajadores: 1) el de quienes no tienen trabajo, 2) el de quienes tienen un trabajo precario 3) el de quienes tienen un trabajo fijo, es decir a la totalidad del mercado de trabajo. 
Afecta en primer lugar a quienes no tienen trabajo, que ven disminuida su capacidad económica y su valorización social y personal. Afecta en segundo lugar a quienes tienen un trabajo inestable que no proporciona ninguna seguridad sobre su duración, de forma que la frontera entre paro y empleo está continuamente en un equilibrio precario. Afecta igualmente a quienes tienen un trabajo fijo, pero que en la situación actual de inseguridad empresarial temen continuamente la aparición de problemas empresariales que hagan inviable la empresa. El miedo al desempleo se ha convertido en una característica general del mercado de trabajo actual.

Son múltiples los datos que indican que el estrés del miedo a la pérdida del trabajo puede ser superior al de la misma pérdida del trabajo ${ }^{91}$. La incertidumbre que acompaña a la anticipación del problema puede hacer más daño que el mismo problema. Los estudios sobre la incertidumbre contractual y sus repercusiones negativas sobre la salud son múltiples. Un estudio reciente analiza la relación entre inseguridad contractual y problemas de salud en 16 países europeos ${ }^{92}$. Los problemas que suelen acompañarle son de todo tipo.

\section{CONCLUSIONES}

Los factores y los riesgos psicosociales no son un tema secundario en la Salud Laboral. En la actual situación organizacional y del mercado del trabajo son uno de sus grandes problemas. Los riesgos de seguridad, ambientales y ergonómicos no han sido solucionados de forma suficiente, y es importante atender a los nuevos riesgos emergentes en estos campos, lo que constituye un verdadero reto para la Salud Laboral (http:// riskobservatory.osha.eu; http://osha.europa.eu/publications/reports/6805478). La gran ventaja que tiene la lucha contra ellos es que nadie discute su importancia y que las formas de intervención suelen ser más claras y precisas. Sin embargo, los factores y riesgos psicosociales, íntimamente vinculados al cambiante mundo del trabajo http:// osha.europa.eu/publications/reports/205), son esquivos en su definición y manejo, y suelen afectar al núcleo de la misma organización. Dado que su naturaleza es dinámica y cambiante, más importante incluso que el esfuerzo directo contra cada uno de ellos es crear una cultura organizacional de la Salud Laboral ${ }^{93}$ que actúe con criterios de alerta, evaluación e intervención.

La globalización y sus productos han creado una enorme área de desregulación económica y laboral que ha profundizado la desigualdad entre sociedades y personas, produciendo un aumento del deterioro social, público y sanitario ${ }^{94}$. La desregulación no ha estado nunca detrás de los progresos en salud, y la desigualdad no ha favorecido en ningún momento el progreso del bienestar, del laboral todavía menos. En este proceso, la recuperación exige una acción política que implique una valorización de lo público, social y organizacional. Hoy día, la elaboración de una ciencia integral de la Salud Laboral $^{95}$ implica conocer los efectos de la organización del trabajo sobre la Salud Laboral, lo que exige a su vez el desarrollo de una cultura de la salud organizacional. De alguna forma, factores y riesgos psicosociales son eventos centinelas de la salud global de una empresa, pues son ellos los que marcan la pauta de la atención y valoración de las empresas hacia las personas.

Los datos actuales no son reconfortantes. Como ha escrito recientemente en su obra póstuma el historiador Tony Judt "Algo va mal" 96 haciendo historia del pasado y del presente, el panorama del futuro no es alentador. Por primera vez en los últimos cincuenta años de historia, los padres temen que sus hijos vayan a tener un mundo peor que el suyo, y parte de ese miedo proviene del incierto mundo laboral y sus riesgos. 


\section{REFERENCIAS BIBLIOGRÁFICAS}

1. Benavides,F., Ruiz-Frutos,C. y García, A. Trabajo y Salud. En F.G. Benavides, C. Ruiz-Frutos y A.M. García (Eds), Salud Laboral. Conceptos y técnicas para la prevención de riesgos laborales. Barcelona: Masson; 2000

2. Pia Chirinos, M. Claves para una antropología del trabajo. Pamplona: EUNSA; 2006.

3. Veyne, P. El Imperio Romano. En Ph. Aries, y G. Duby, (Eds). Historia de la vida privada. V. I. Madrid: Taurus; 1990;

4. Judt, Tony. Postguerra. Una historia de Europa desde 1945. Madrid: Taurus; 2010.

5. EWCS. Fourth European Working Conditions Survey. European Foundations for the improvement of living and working conditions: Dublin. 2007;

6. Bernabeu, J; Perdiguero,E. y Zaragoza,P. Desarrollo histórico de la salud laboral. En F.G.,Benavides, Ruiz-Frutos,C. y García, A.M. (Eds). Salud Laboral. Conceptos y técnicas para la prevención de riesgos laborales. Barcelona. Masson ; 2000.

7. Hobsbawm, E. La era del capital. 1848-1875. Crítica: Barcelona; 2011.

8. Pound, N.J.G. La vida cotidiana. Historia de la cultura material. Crítica: Barcelona; 1999.

9. Moreno-Jiménez, B y Garrosa Hernández, E. Globalización y riesgos laborales emergentes. Editorial. Ciencia \& Trabajo, 2009, 11, 32, 31-35

10. González Maestre, D. Ergonomia y Psicosociologia. Madrid: Confemetal; 2003.

11. Llaneza Alvarez, F. J. Ergonomía y Psicosociologia Aplicada. Valladolid: Lex Nova; 2003.

12. OIT Factores psicosociales en el trabajo: reconocimiento y control. Ginebra: OIT;1986.

13. OIT. Enciclopedia de la Seguridad y la salud en el Trabajo. Madrid: Ministerio del Trabajo y Asuntos Sociales; 1998.

14. Cooper, C y Williams, S. Creating Healthy Work Organizations. Chichester: Wiley; 1994

15. Minztberg, H. Structure in fives: designing effective organizations. Englewood Cliff,N.J.: Prentice Hall; 1993

16. Kalimo, R., El-Batawi, M., \& Cooper, C. L. Los factores psicosociales en el trabajo y su relación con la salud. Ginebra: OMS; 1988

17. Benavides, F., Gimeno, D., Benach.J., Martinez, J. M., Jarque, S., \& Berra, A. Descripción de los factores de riesgo psicosocial en cuatro empresas. Gac Sanit, 2002, 16,3, 222-229.

18. Cox, T. \& Griffiths, A. J. The assessment of psychosocial hazards at work. En M.J.Schabracq, J. A. M. Winnubst, \& C. L. Cooper (Eds.), Handbook of Work and Health Psychology. Chichester: Wiley and Sons; 1996. p. 127-146.

19. Taris, T. \& Kompier, M. Job demands, job control and learning behavior: Review and research agenda. En: Antoniou, A. \& Cooper, C. (Eds.) Research companion to Organizational Health Psychology, Cheltenham: Edward Elgar Publishing LTD; 2005. p. 32-150.

20. Cooper, C. L. Theories of Organizational Stress. Oxford: Oxford University Press; 1998

21. Cooper, C. L., Dewe, Ph., \& O'Driscoll, M. P. Organizational Stress. Thousand Oaks: Sage Publications; 2001.

22. Selye, H. Tensión sin angustia. Madrid: Guadarrama; 1975.

23. Lazarus, R. \& Folkman, S. Estrés y Procesos cognitivos. Barcelona: Martinez Roca; 1986

24. Hobfoll, S. E. Conservation of Ressources. A new attempt of conceptualizing stress. American Psychologist, 1989, 44, 3, 513-524.

25. Mc Ewen, B. The end of the stress as we know it. Washington: Joseph Henry Press; 2002.

26. European Social Partners. Framework Agreement on Work-related Stress. Brussels: European social partners - ETUC, BUSINESSEUROPE, UEAPME and CEEP; 2004.

27. European Social Partners Framework Agreement on Harassment and Violence at Work. Brussels: European social partners - ETUC, BUSINESSEUROPE, UEAPME and CEEP; 2007.

28. Leka, S., Jain, A., Zwetsloot, G., Vartia, M., \& Pahkin, K. Psychosocial risk management: the importance and impact of policy level interventions. En S. Leka \& T. Cox (Eds.), The european framework for psychosocial risk management. PRIMA-F. Nottinghan.: I-WHO; 2008. p. 115-135.

29. Brunet, L. El clima de trabajo en las organizaciones. México: Trillas; 1987

30. Leka, S. Jain,A. Health Impact of Psychosocial hazards at work: An overview. Brussels: WHO; 2010 
31. INSHT. III Encuesta Nacional de Condiciones de Trabajo. Madrid: Ministerio de Trabajo y Asuntos Sociales; 1999

32. INSHT VI Encuesta nacional de Condiciones de trabajo. Madrid: Ministerio de Trabajo y Asuntos Sociales.

33. Collier, P. El club de la miseria. Barcelona. De Bolsillo; 2010

34. Kaufmann, D. y Kraay, A. "Growth without governance," Policy Research Working Paper Series ,2928, The World Bank; 2002.

35. Hobsbawm, E. Historia del Siglo XX. Barcelona: Crítica; 2003.

36. Sennett, R. El declive del hombre público. Barcelona: Península; 2002.

37. Lyon, D. Postmodernidad. Madrid: Alianza;1996.

38. Lyotard, J.F. La condicion postmoderna. Madrid: Cátedra;1989.

39. Drucker, P.F. La sociedad postcapitalista. Barcelona: Apóstrofe;1995.

40. Harvey, D. La condición de la postmodernidad. Investigación sobre los orígenes del cambio actual. Buenos Aires: Amorrortu; 2008.

41. Toffler. A. La Empresa Flexible . Barcelona: Plaza Janes; 1990.

42. Carnoy, M. El trabajo flexible en la era de la información. Madrid: Alianza; 2001.

43. Handy, Ch. Más allá de la certidumbre. Los cambiantes mundos de las organizaciones. Barcelona: Apóstrofe; 1997.

44. Comin, F. Revolución industrial. Capitalismo. En M. Artola (Ed). Historia de Europa. Vol II. Madrid: Espasa; 2007. p. 373-463.

45. De Luis Martín, F. La segunda revolución Industrial y sus consecuencias. En M. Paredes (Ed), Historia Universal contemporánea. Vol. I. Barcelona: Ariel; 2009, p 197-228.

46. Becker, G.S. Human Capital. Princeton University Press: Princeton, N.J; 1964.

47. Casado, J.M. El valor de la persona. Nuevos principios para la gestión del capital humano. Madrid: Prentice Hall; 2003.

48. Bonache, J. y Cabrera, A. Dirección estratégica de personas. Evidencias y perspectivas para el siglo XXI. Madrid: Prentice Hall; 2002.

49. Sennet, R. El respeto. Sobre la dignidad del hombre en un mundo de desigualdad. Barcelona: Anagrama; 2003.

50. Senge, P. M. La quinta disciplina. Barcelona: Granica, 1997.

51. Ashkanasy, N.M. , Hartel, Ch. E.J. y Zerbe, W.J. Emotions in the workplace. London: Quorum Books; 2000

52. Goleman, D. y Cherniss, C. Inteligencia emocional en el trabajo. Barcelona: Kairós; 2005.

53. Luthans, F., Youssef, C.M., y Avolio, B.J. Psychological Capital. Developing the human competitive edge. Oxford: Oxford University Press; 2007.

54. Rodríguez-Carvajal, R Moreno-Jiménez, B., Rivas-Hermosilla S , Álvarez-Bejarano, A and Sanz Vergel, A. Positive Psychology at Work: Mutual Gains for Individuals and Organizations. Revista de Psicología del Trabajo y de las Organizaciones, 2010, 26, 3, 235-253.

55. Robbins, St. y Judge, T.A. Comportamiento organizacional. Madrid: Prentice Hall; 2009.

56. Luthans, F. Comportamiento Organizacional. Madrid: McGraw Hill; 2008.

57. Finkel, L. La organización social del trabajo. Madrid: Pirámide; 1994.

58. Elizur, D., y Shye, S. "Quality of work life and its relations to quality of life", Applied Psychology: An International Review, 1990, 39, 275-291.

59. Moreno Jiménez, B. y Ximenez ,C. La evaluación de la calidad de vida. En G. Buela., V. Caballo y J.C. Sierra (Eds.) Manual de Evaluación en psicología Clínica y de la salud. Madrid: Ed Siglo XXI; 1996, p. 1045-1070.

60. Mohn, R. La responsabilidad social del empresario. Madrid: Círculo de lectores; 2005.

61. Perdiguero, T.G. La responsabilidad social de las empresas en un mundo global. Madrid: Anagrama; 2003.

62. Thomas, G. y Novak, M. Corporate Social Responsibility: A definition. Graduate School of Business: Curtin University of Technology; 2006. 
63. Elkington, J. y Hartigan P. El poder de la locura. Empresas rentables que cambian el mundo. Barcelona: Ediciones Deusto; 2008

64. Martínez, J.L., Simón, C. Agüero, A. La acción social de la empresa. Madrid: Prentice; 2003.

65. Henry, J. The Healthy organization. En A.G. Antoniou y C.L. Cooper (Eds). Research Companion to organizational Health Psychology.. Cheltenham,UK: Edward Elgar; 2005, p. 383-392.

66. Beck, U. La sociedad del riesgo. Hacia una nueva modernidad. Barcelona: Paidós; 2006.

67. Beck, U. La sociedad del riesgo mundial. Em busca de la seguridad perdida. Barcelona: Paidós; 2008.

68. OIT Riesgos emergentes y nuevos modelos de prevención. Ginebra: OIT; 2010

69. OSHA European Agency for Safety and Health at Work. EUROPEAN RISK OBSERVATORY REPORT. Expert Forecast on emerging Psychosocial risks related to occupational safety and health; 2007. osha. europa.eu/en/publications/reports/7807118.

70. OSHA (2010) European Agency for Safety and Health at Work. EUROPEAN RISK OBSERVATORY REPORT. European Survey of Enterprises on New and Emerging Risk; 2010 osha.europa.eu/en/ publications/reports/esener1_osh_management

71. Bell, D. El advenimiento de la sociedad industrial. Madrid: Alianza; 1994.

72. Garrido, L. y González, M J.J. Mercado de trabajo, ocupación y clases sociales. En J.J. González y M. Requena, (Eds). Tres décadas de cambio social en España. pp 81-126. Madrid: Alianza; 2005.

73. Gutierrez, R. Trabajo y ocupaciones. En S. del Campo y J.F. Tezanos (Eds). La sociedad.. Madrid: Biblioteca Nueva; 2008, p. 321-352

74. Hochschild, A. R. The managed heart: Commercialization of human feeling. Berkeley, CA: University of California Press. Wiley; 1983.

75. Martínez-Iñigo, D. Evolución del concepto de Trabajo Emocional: dimensiones antecedentes y consecuencias. Una revisión teórica. Revista de psicología del Trabajo y de las Organizaciones, 2001, 17, 2, 131-153

76. Moreno-Jiménez, Bernardo, Galvez Herrer, Macarena, Rodríguez Carvajal, Raquel, Garrosa Hernández, Eva. Emociones y salud en el trabajo. Análisis del constructo de "Trabajo Emocional" y propuesta de evaluación. Revista Latinoamericana de Psicología, 2010, 42,1, 63-74.

77. Richards, J.M. \& Gross, J.J. Composure at any cost? The cognitive consequences of emotion suppression. Personality and Social Psychology Bulletin, 1999, 25, 8, 1033-1044.

78. Wong, Y.J., Pituch, K.A. \& Rochlen, A.B.. Men's restrictive emotionality: An investigation of associations with other emotion-related constructs, anxiety and underlying dimensions. Psychology of Men \& Masculinity, 2006, 7, 2, 113-126.

79. Giddens, A. Sociologia. Madrid: Alianza; 2005.

80. Beck, U. ¿Qué es la globalización.? Barcelona: Paidós; 2008

81. Stiglitz, J.E. El malestar en la globalización. Madrid: Punto de lectura; 2006.

82. Friedman, Th. La tierra es plana. Madrid: Martínez Roca; 2006.

83. Ziegler, J. Los nuevos amos del mundo. Barcelona: Destino; 2006

84. Klein, N. NO LOGO. Madrid: Paidós; 2002.

85. Castillo, J.J. La soledad del trabajador globalizado. Madrid. Los libros de la catarata; 2008.

86. Zozaya González, N. Las fusiones y adquisiciones como forma de crecimiento empresarial. Madrid. Ministerio de Trabajo; 2007.

87. Jiménez Jiménez, C., y Sánchez Laguna, J.L. Análisis del downsizing (reducción de plantilla) como fenómeno psicosocial. Revista de Psicología del Trabajo y de las Organizaciones, 2005, 21, 3, 181-206

88. Benavides, F., Delclos, J., Benach. J. y Serra, C. Lesiones por accidentes de trabajo. Una prioridad en atención pública. Rev. Esp. Salud Pública, 2006, 80, 5, 553-565.

89. Arias, X.C. y Costas, A. La torre de la arrogancia. Políticas y mercados después de la tormenta. Barcelona: Ariel; 2011.

90. Beck, U. Un nuevo mundo feliz. La precariedad Del trabajo en La era de la globalización. Barcelona: Paidós; 2000

91. Hartley, J. Models of job insecurity and copping strategies of organizations. En J.E. Ferrie, M.G.Marmot y J. Griffiths (Ed.),Labour market changes and job insecurity: a challenge for social welfare and health promotion Copenhagen, Dinamarca: World Health Organization; 1999, p.127-150.

92. Laszlo, K., Pikhart, H., Kopp., M., Bobak, M., Pajak, A., Malyutina, S., Salavecz, G. \& Marmot, M. Job insecurity and health: A study of 16 European countries. SOC SCI MED, 2010, 70, 6, 867-874. 
93. Wilderom, C.P.M. Toward positive work cultures and climates. En N. Ashkanasy C.P.M, Wilderom M.F. Peterson (Eds), The Handbook of Organizational Culture and Climate New York: Sage; 2010, p 79-84.

94. Wilkinson, R. y Pickett, K. Desigualdad. Un análisis de la infelicidad colectiva. Madrid: Turner; 2009.

95. Lundberg, U. y Cooper, C.L. The Science of Occupational Health. Oxford: Wiley-Blackwell; 2011.

96. Judt, T. Algo va mal. Madrid: Taurus; 2010.

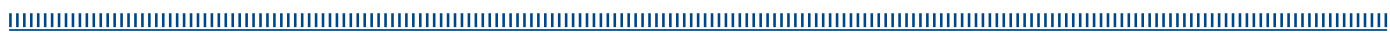

